\title{
VELARDE LOMBRAÑA, J. TEORÍA DE LA DEFINICIÓN DE LEIBNIZ, GRANADA: COMARES, 2015, 119 PÁGS.
}

\section{RESEÑA: Miguel Escribano \\ Universidad de Granada}

La colección Nova Leibniz (http://leibniz.es/novaleibniz.htm) es fruto de la colaboración entre el proyecto de investigación "Leibniz en español" (www.leibniz.es) y la Editorial Comares. El proyecto "Leibniz en español", coordinado por el catedrático de Filosofía de la Universidad de Granada, Juan Antonio Nicolás, lleva ocho años implicado en la edición en esta misma editorial de una selección sistemática de las obras de G.W. Leibniz. Entre los éxitos del proyecto cabe destacar además la constitución de la Red Iberoamericana Leibniz, cuyo principal objetivo es coordinar, visibilizar y potenciar el trabajo realizado por los investigadores de habla española y portuguesa, y la creación de la Biblioteca Hispánica Leibniz (www.bibliotecahispanicaleibniz.es), donde se está reuniendo todo lo publicado de y sobre Leibniz en el ámbito cultural de las lenguas española y portuguesa.

El objetivo de la colección Nova Leibniž es publicar trabajos de investigación novedosos relativos al pensamiento y la obra de G.W. Leibniz. Se presenta como una colección dirigida a la comunidad investigadora internacional, aceptando trabajos originales e inéditos escritos en alguno de los idiomas siguientes: español, portugués, inglés, francés, alemán o italiano. Entre 


\section{Dossiê Leibniz, Dissertatio - Volume Suplementar 03 | UFPel [2016]}

sus últimas novedades cabe destacar: Deo volente. El estatus de la voluntad divina en la Teodicea de Leibniz, de M. Griselda Gaiada y Curvas y espejos. El carácter funcional de la actividad monádica en G.W. Leibnir, de Laura Herrera.

El profesor Julián Velarde Lombraña, catedrático de Filosofía en la Universidad de Oviedo, ha dedicado su larga y fructífera trayectoria al estudio de la lógica y su historia, la teoría del conocimiento y la teoría del lenguaje. Ocupa un lugar destacado sus traducciones y trabajos sobre Platón, Aristóteles, Tomás de Aquino, Caramuel, Leibniz y Peano. Es precisamente su labor de investigación sobre el pensamiento del filósofo de Hanover la que ahora nos gustaría reseñar.

Además de su magnífica edición en castellano de la Monadología (Madrid: Biblioteca Nueva, 2001) y la publicación de numerosos y reconocidos artículos, su entrega a la obra y el pensamiento de Leibniz ha dado en los últimos años dos frutos destacados. En el año 2013 la Editorial Comares publicaba el volumen que para la edición de las Obras filosóficas y cientificas de G.W. Leibniz había preparado junto a Leticia Cabañas, dedicado a los textos sobre Lengua Universal, Característica y Lógica (Granada: Comares, 2013, 528 págs.). Paralelamente a la preparación de esta edición, el profesor Julián Velarde ha ido desarrollando una complementaria labor de investigación e interpretación sobre el pensamiento leibniziano que fue apareciendo en una serie de trabajos y ponencias en congresos. Toda esta labor culminó con la publicación del libro que aquí presentamos, Teoría de la definición de Leibniz (Granada: Comares, 2015), 
que recomendamos leer acompañado de la obra anterior, pues la mayoría de los textos sobre los que Velarde construye su interpretación están recogidos en esa edición.

La teoría de la definición es un elemento nuclear del pensamiento leibniziano dedicado a la búsqueda progresiva de un orden y una sistematización de todos los conocimientos, proyecto al que el filósofo dio el nombre de Ciencia General. Este método de sistematización se funda en el principio leibniziano de la ligazón universal de todas las cosas, que conecta con una concepción de la razón donde la inserción y el análisis de cada concepto o noción obedece a leyes generales que nos permiten, y obligan, a transitar analógicamente a largo de los distintos ámbitos del conocimiento.

La idea de Leibniz es que si conseguimos establecer el conocimiento sobre la base de unas nociones primitivas podremos formalizar y describir todos los reinos del saber. Todas las derivas que dio este proyecto se pueden sintetizar en torno a la problemática naturaleza de estas nociones primitivas, desde sus comienzos con la Combinatoria y la Característica hasta sus últimos logros a partir del descubrimiento del Cálculo.

Al igual que en su física Leibniz rechazó el atomismo en cuanto comprendió que en la naturaleza todo cuerpo está actualmente dividido al infinito, en su teoría de la definición Leibniz terminó por desechar la idea de encontrar una noción que una vez bien definida actuara como un átomo, dado que, como pronto comprendió, el análisis de una noción es inagotable. 


\section{Dossiê Leibniz, Dissertatio - Volume Suplementar 03 | UFPel [2016]}

Este "percance" relativo a la naturaleza de las nociones primitivas hizo por otra parte avanzar al proyecto leibniziano. En un primer momento Leibniz desdobló su metodología, bien estemos tratando con verdades necesarias (de análisis finito), bien con verdades contingentes (donde el análisis es infinito). En la metafísica leibniziana esta distinción entre necesidad y contingencia opera ligada a la distinción entre posible y existente, relación donde queda trabada la diferencia y la articulación entre los principios de la razón: principios lógicos y principios ontológicos. De todo ello deriva la distinción que Leibniz hace entre definición nominal y definición genética. Una muestra de cómo opera en su distinción y articulación este desdoble del análisis lo encontramos en la posición leibniziana con respecto a la prueba de la existencia de Dios, a la que Velarde dedica el tercer capítulo del libro. Leibniz se desmarca tanto de la definición cartesiana de Dios como causa sui como de la definición genética de Spinoza por el mismo motivo: confundir razón y causa. Leibniz reinterpreta esta distinción desde su teoría de los requisitos, sobre la que Velarde se extiende en el primer capítulo.

El análisis de términos, si se lleva a cabo adecuadamente bajo lo guía de los principios de no contradicción y de identidad, nos ha de conducir a la definición nominal, que nos permite enumerar las notas o requisitos suficientes para distinguir una cosa de todas las demás. Aunque en la definición nominal esté cifrada la constitución de la cosa (sus requisitos internos) lo más útil, afirma Leibniz, es si sus requisitos incluyen además la generación de la cosa, y nos 
ofrecen una definición genética. La primera, la definición nominal, es capaz de denotar la esencia de la cosa y la segunda, la definición genética, además demostrar que de ésta (de la esencia) se sigue la existencia: la definición genética, afirma Leibniz, envuelve una causa próxima de algo. Leibniz divide la definición en nominal y genética en función de que los constituyentes de la definición, esto es, los requisitos, sean o bien internos, y entonces representen la razón suficiente de la posibilidad de una cosa, o bien externos, y entonces incluyan además su causa. El éxito de la teoría de la definición radica en conseguir mostrarnos cómo se correlacionan los requisitos internos y los requisitos externos, sin confundirlos, como según Leibniz hacían Descartes y Spinoza. Para avanzar en ello Leibniz insertará ingredientes procedentes del Cálculo y la Dinámica.

A partir de aquí se abren varias opciones. La tomada por Julián Velarde en su libro es quizás una de las más interesantes, dado que permite abarcar más aspectos del pensamiento leibniziano. Se trata de saltar de la teoría de la definición a la teoría de la sustancia. Leibniz nos facilita ese paso, dada la relación intrínseca que establece entre los principios lógicos y los ontológicos. Como nos muestra Velarde, para comprender el sustrato que nutre la concepción leibniziana de la sustancia como noción completa o como ley de la serie hay que continuar el tratamiento leibniziano de las relaciones entre posibilidad-necesidad y esencia-existencia.

La distinción entre definición nominal y genética nos lleva a un doble tratamiento sobre lo posible. Por un lado, nos dice Velarde, nos encontramos 


\section{Dossiê Leibniz, Dissertatio - Volume Suplementar 03 | UFPel [2016]}

con la esfera de las esencia posibles, que son eternas y necesarias y que existen desde la eternidad en el entendimiento divino. Lo posible-esencial constituye el objeto interno del entendimiento divino desde el que Dios produce, actualiza, crea o da existencia a las cosas o verdades contingentes. En tanto verdades eternas, los posibles-esencias son independientes de la acción divina y sólo obedecen al principio de no contradicción. Nos dice así Velarde que la posibilidad de lo absolutamente posible y la verdad de las verdades eternas están más allá, y son independientes de, la voluntad, el poder y el entendimiento de Dios, lo que vincula a Leibniz con la tradición platónica. Ilustra Velarde esta posición de Leibniz con los comentarios que el filósofo de Hanover escribió sobre el Leptotatos de Caramuel.

Por otro lado, distinguimos un segundo tipo de posibles: los posibles realizados o existentes, sobre los que rige otra serie de principios.

(1) El principio de perfección o de máxima esencia nos define lo posible como categoría ontológica: lo posible demanda existencia en función de su grado de perfección o esencia. La existencia no es por tanto un complemento de la esencia sino que está contenido y determinado por ella. Nos conduce este principio hasta la teoría de la composibilidad (que enfrenta a Leibniz con la tesis spinozista de que todos los posibles existen) y a una lógica posibilista, cuya origen, nos muestra Velarde, lo podemos remontar hasta Aristóteles.

(2) El segundo principio es el de razón suficiente. Como el anterior, este principio atañe a la existencia o no existencia de todo ente, pero además, a 
su forma de ser, esto es, al conjunto de los atributos que lo definen como existente, para los cuales ha de haber una razón determinante que no es necesitante: todo ente se encuentra necesariamente en la serie de lo existente según alguna razón, pero no es necesario el que tenga está razón u otra. Enlaza Velarde desde este principio con la teoría de la sustancia como noción completa (unidad que incluye o envuelve todos sus atributos como existente) y con la interpretación de la naturaleza de la sustancia como ley de la serie (razón de la serie de esos atributos).

A la concepción de la naturaleza de la sustancia como ley de la serie están dedicados los dos últimos capítulos del libro, el cuarto, centrado en la sustancia finita, y el quinto capítulo sobre Dios. En esta última parte del libro es donde la interpretación de Velarde se muestra más original. Primero, en el modo como articula las ideas del Cálculo y la Dinámica para pensar la naturaleza activa de la sustancia en términos de la razón o forma que da unidad y orden al desarrollo de una serie. Resulta iluminador respecto a la concepción leibniziana de la sustancia el modo como Velarde aplica el modelo serial para comprender caracteres fundamentales de su naturaleza, como son la unidad y la actividad. Destacar aquí la complicidad de Velarde con la investigación de Laura Herrera sobre el funcionalismo leibniziano (publicada en esta misma colección). Velarde va más allá de Herrera al extender el modelo de la serie a la explicación de la arquitectónica subyacente a la visión del mundo de Leibniz, desde el mínimo al máximo orden de perfección, desde las simples mónadas desnudas a los 


\section{Dossiê Leibniz, Dissertatio - Volume Suplementar 03 | UFPel [2016]}

espíritus, o desde la naturaleza representativa de los sujetos al entendimiento infinito de Dios. Velarde nos muestra además cómo se correlacionan todos los principios leibnizianos en esta arquitectónica visión del mundo y cuál es el papel de Dios en todo ello.

Partiendo de la distinción que hace Leibniz entre homogeneidad y homogonía (por ejemplo, para explicar la relación entre el punto y la línea y la conversión entre ambos), Velarde nos dice que Leibniz se habilita una vía para explicar lo continuo desde lo discontinuo, como un caso de progresión que permite que una cosa llegue a ser (o se desvanezca en) una cosa de un género distinto, sin perder por ello los caracteres que la caracterizan (lo que explica, por ejemplo, que la línea esté compuesta de puntos). Esta interpretación de la ley de la continuidad leibniziana en términos de homogonía nos muestra como el mundo de los posibles existentes, mediante una progresión serial continua de más a más posibilidad, en el límite, esto es, en el máximo de posibilidad, se desvanece en el ser necesario, en Dios. En el infinito, nos dice Velarde, la posibilidad o la esencia máxima viene a equivaler a la necesidad o la perfección máxima, esto es, a Dios, definido como límite de la serie infinita de los posibles. 